\title{
Advantages of Using Additive Manufacturing to Build "Green" Fuels for Hybrid Propulsion
}

\author{
Stephen A Whitmore* \\ Mechanical and Aerospace Engineering Department, Utah State University, USA
}

\begin{abstract}
Advantages achieved by 3-D printing of hybrid rocket fuels are presented. This approach overcomes multiple manufacturing issues frequently associated with hybrid rockets including low fuel regression rates and poor ignitability. Using 3-D printing also allows fabrication of fuel grains with port shapes that enhance burn properties and increase volumetric efficiency. Printing ABS creates small, micronthick features that provide surface arc paths allowing fuel pyrolysis at moderate voltages. When electrodes are embedded into the material and the arc occurs simultaneously with the introduction of oxidizer flow, the vaporized fuel "seeds" combustion. This concept has been developed into a power-efficient ignition system allowing hybrid motors to be started, stopped, and restarted with a high level of reliability. Several small spacecraft system prototypes with multiple restart capabilities have been demonstrated. Static tests performed under low altitude ambient conditions exhibit delivered specific impulse exceeding $228 \mathrm{~s}$. These values are greater than nominally achieved by hydrazine under vacuum-conditions. Typical ignition sequence required input power is less than 5 $\mathrm{W}$-s. The delivered specific output power exceeds $5 \mathrm{~kW} / \mathrm{g} / \mathrm{s}$. Data from tests with helical fuel ports demonstrated fuel regression rate increases by a factor of three. This emerging "green" technology promises to replace hydrazine for many applications.
\end{abstract}

\section{Keywords}

Hybrid-propulsion, Small-spacecraft, Additive-manufacturing, Green-propellants, Environmental-sustainability, Low-hazard, Arc-ignition, Fuel regression-rate

\section{Introduction}

Hydrazine (N2H4) has been the standard for in-space propulsion for more than 50 years and is by far the most commonly used monopropellant for primary spacecraft propulsion and attitude control thrusters. Hydrazine thrusters are simple, versatile, and dependable. Hydrazine is attractive as a spacecraft monopropellant because of its high hydrogen content. When passed through a catalyst the decomposition produces ammonia, nitrogen, and hydrogen gas. The reaction is highly exothermic and associated gaseous products reach temperatures of exceeding $1300^{\circ} \mathrm{C}$. Under realistic operating conditions hydrazine decomposition can produce specific impulse levels exceeding 220 seconds under vacuum conditions [1,2].
Unfortunately, hydrazine is a powerful reducing agent that poses serious biological and environmental concerns. Hydrazine is extremely destructive to living tissues, and is a known human carcinogen. The US Environmental Protection Agency (EPA) classifies hydrazine as "highly toxic". Hydrazine possesses a high vapor pressure and boils readily at room temperature. Thus, there exists significant risk of respiratory contamination and special servicing procedures that employ full-pressure Self-Contained Atmospheric Protective Ensemble (SCAPE) suits are required [3].

Although procedures are in place to allow hydrazine to be managed safely on tightly controlled military reservations and at government-operated launch facil-

${ }^{*}$ Corresponding author: Stephen A Whitmore, Mechanical and Aerospace Engineering Department, Utah State University, 4130 Old Main Hill, UMC 4130, Logan, Utah, USA, E-mail: Stephen.Whitmore@usu.edu

Received: January 27, 2016: Accepted: March 31, 2017: Published: April 03, 2017

Copyright: (C) 2017 Whitmore SA. This is an open-access article distributed under the terms of the Creative Commons Attribution License, which permits unrestricted use, distribution, and reproduction in any medium, provided the original author and source are credited. 
ities; the toxicity and explosion potential of hydrazine requires extreme handling precautions that increase operational complexity. Increasingly, with a growing regulatory burden, infrastructure requirements associated with hydrazine transport, storage, servicing, and clean up of accidental releases are becoming cost prohibitive [4,5].

A recent study [6,7] by the European Space Agency Space Research and Technology Center (ESTEC) has identified two essential design elements to achieving low cost commercial space access and operations; 1) Reduced production, operational, and transport costs due to lower propellant toxicity and explosion hazards, and 2) Reduced costs due to an overall reduction in subsystems complexity and overall systems interface complexity. The ESA/ESTEC study showed the potential for considerable operational cost savings by simplifying propellant ground handling procedures. Developing a non-toxic, stable "green" alternative for the most commonly used in-space propellant-hydrazine-was highly recommended.

In response to this recommendation, for the past decade the US Air Force (USAF) and the Swedish Space Corporation (SSC) subsidiary Ecological Advanced Propulsion Systems (ECAPS) have been pursuing less hazardous alternatives to hydrazine [8,9]. The two most highly-developed "green-propellant" alternatives are based on aqueous solutions of the ionic liquids (Ils) Ammonium Dinitramide (ADN) [10], and Hydroxylamine Nitrate (HAN) [11].

However, both of the above-mentioned IL-propellants are toxic to organic tissue, and special handling precautions are required. IL-based propellants are not truly "green." Thus, the USAF has recently begun to refer to such IL-formulations more properly as having "reduced toxicity" properties.

Ionic liquids are water-soluble ammonium-salts that normally exist in solid form at room temperature, but melt below the boiling point of water. When dissolved in water these materials exhibit very strong ion-to-ion interactions. The surface charge attractions of these ions act to tightly hold them together in solution, and as a result produce a significantly lowered vapor pressure [12]. This lowered vapor pressure reduces the risk of toxicity posed by any escaped vapor from the monopropellant containment vessel. Spacecraft may be serviced without the use of SCAPE suits [13].

In August 2011, ECAPS announced the results of a year-long series of in-space tests of a 1-N thruster comparing their High Performance Green Propellant (HPGP) Brand Name LMP-103S, to hydrazine on the Prisma spacecraft platform. ECAPS claims that HPGP delivered equivalent-to-superior performance when compared to mono-propellant hydrazine [14]. NASA has recently selected the USAF-developed HAN-based propellant AF-M315E for its "Green Propellant Infusion Mission (GPIM) [15].

Unfortunately, in additional to potential toxicity, there exist several key developmental issues associated with IL-based propellants that make them unsuitable for small spacecraft applications. The high water content makes IL-propellants notoriously hard to ignite. Multiple catalyst systems have been developed to augment IL ignitability; however, room temperature ignition does not currently exist. Catalyst beds must be preheated from $350-400{ }^{\circ} \mathrm{C}$ before and during ignition, and this preheat can consume up to 15,000 joules of energy. Catalyst beds and associated heating systems add significantly to the inert mass of the spacecraft and the high-wattage preheat requirement presents a significant disadvantage for small spacecraft where power budgets are extremely limited.

Finally, due to very slow reaction kinetics [16] at moderate pressures $(2000-3000 \mathrm{kPa})$ system latencies associated with IL-based propellants are significant for moderate chamber pressures and may limit the usefulness of IL-propellants for reaction control systems.

Clearly, significant technology improvements must occur before IL-based systems can be employed as a primary propulsion unit or as part of the reaction control system for small spacecraft. With the current state of propulsion technology, the only proven non-hazardous propulsion alternative to hydrazine, and available for small ride-share payloads, is based on low-performing coldgas thrusters.

\section{Hybrid Rocket Systems as a "Green" Space Propulsion Alternatives}

When compared to conventional liquid- and solid-propelled rocket systems, hybrid rockets-where the propellants typically consist of a benign liquid or gaseous oxidizer and an inert solid fuel-possess well-known operational safety and handling-advantages. A study by the U.S. Department of Transportation concluded that hybrid rocket motors can be safely stored and operated without a significant risk of explosion or detonation, and offer the potential to significantly reduce operating costs for commercial launch vehicles [17].

Hybrid rocket systems can offer higher specific impulse performance than monopropellant hydrazine-based systems; and their inherent-design safety offers a significant potential for ride-share spacecraft applications. However, in spite of these well-known safety and handling advantages; conventionally-designed hybrid rocket systems have not seen widespread commercial use due to several key drawbacks that exist with conventional hybrid-system designs. 


\section{Disadvantages with the current state-of-the-art in} hybrid rocket system

First, the internal motor ballistics of hybrid combustion produce fuel regression rates that are typically 25\%-30\% lower than solid fuel motors in the same thrust and impulse class [18]. These lowered fuel regression rates tend to produce unacceptably high Oxidizer-to-Fuel $(\mathrm{O} / \mathrm{F})$ ratios that may lead to combustion instability, erosive burning, nozzle erosion, and reduced motor duty cycles.

To produce lowered $\mathrm{O} / \mathrm{F}$ ratios hybrid fuel grains have been traditionally fabricated with very large length-to-diameter ratios. These longer aspect ratios would produce spacecraft thrusters systems that are difficult to package within launch vehicles, and require additional support structures to accommodate launch loads. Generally, the poor volumetric efficiency, defined as a large surface area for a given internal volume, has made hybrid motors less compatible for spaceflight applications than traditional solid or bi-propellant configurations.

Second, because of the relative propellant stability, hybrid rocket systems can be difficult to ignite; and a substantial ignition enthalpy source is required. The ignition source must provide sufficient heat to pyrolize the solid fuel grain at the head end of the motor, while simultaneously providing sufficient residual energy to overcome the activation energy of the propellants. Such high-energy devices often come with a suite of environmental and objectives risks, and operational challenges.

Most conventional hybrid rocket applications have used high output pyrotechnic or "squib" charges to initiate combustion. Pyrotechnic charges are extremely susceptible to the Hazards of Electromagnetic Radiation to Ordnance (HERO) [19], and large pyrotechnic charges present a significant explosion hazard that is incompatible with rideshare opportunities. Most importantly, for nearly all applications pyrotechnic ignitors are designed as "one-shot" devices that do not allow a multiple restart capability.

Thus, the great potential for re-start able upper stages or in-space maneuvering systems using hybrid propulsion remains largely unrealized. An operational hybrid system with multiple restart capability does not currently exist. The lack of an on demand restart capability has traditionally precluded hybrid motors from being seriously considered for in-space propulsion applications.

Finally, the "cast and cure" methods for producing hybrid fuel grains made from traditional thermosetting solid propellant binder materials like including $\mathrm{Hy}-$ droxyl-Terminated Polybutadiene (HTPB), Polybutadiene Acrylonitrile (PBAN), and Glycidyl Azide Polymer (GAP) are necessarily labor intensive, and high production rates cannot be achieved without a significant manufacturing infrastructure. These binder materials are mixed from liquid base-components, degassed under vacuum, and then cast and cured in a fuel grain mold. This labor intensive manufacture and assembly approach results in market prohibitive production costs and cannot produce the numbers and varieties of motors required to support the what is expected to be a fast-growing commercial space industry.

The isocyanate-based materials used to cure these previously described fuel polymers present a wide variety of Environmental Safety and Occupational Health (ESOH) risks including carcinogenic and detrimental reproductive effects. The US Department of Defense considers these materials to be environmentally unsustainable for large-scale propellant production, and is actively seeking replacement alternatives [20].

\section{Additive manufacturing as a solution to existing hybrid propulsion technology disadvantages}

Whitmore, and Peterson [21] at Utah State University recently investigated the use of additively-manufactured Acrylonitrile Butadiene Styrene (ABS) thermoplastic as a hybrid rocket fuel material. In this research hybrid fuel grains made from both traditionally-cast HTPB and 3-D printed ABS were burned with Nitrous Oxide $\left(\mathrm{N}_{2} \mathrm{O}\right)$ as the oxidizing agent. The test results demonstrated that the performance levels in terms of specific impulse (Isp), characteristic velocity $\left(\mathrm{c}^{*}\right)$, and total specific gravity (sg) of the consumed propellants were nearly identical. Neither fuel material exhibited a statistically significant combustion advantage when compared to the other.

When compared to HTPB, however, ABS has several mechanical properties that are very attractive for hybrid rocket fuel applications. ABS is an inexpensive thermoplastic material that is widely mass-produced for a variety of non-combustion applications including household plumbing and structural materials. ABS is a non-crystalline material and as such does not possess a true melting point. Instead at a temperature of approximately $105^{\circ} \mathrm{C}$, ABS transitions into a highly-softened semi fluid state. This softened state exists over a wide temperature range before the material gasifies.

Additive manufacturing of hybrid propellants: This phase-transition property makes ABS the material of choice for a modern form of additive manufacturing known as Fused Deposition Modeling (FDM). In FDM, a plastic filament is unwound from a coil and supplies material to an extrusion nozzle. By industry standards the nozzle is heated approximately $230^{\circ} \mathrm{C}$ to soften the material sufficiently to allow precise extrusion. The motion of the nozzle in three-dimensions is directed by a Computer Numerically Controlled (CNC) mechanism. Identical pieces can be produced simultaneously by multiple vendors using a well-developed commercial technology. Because 


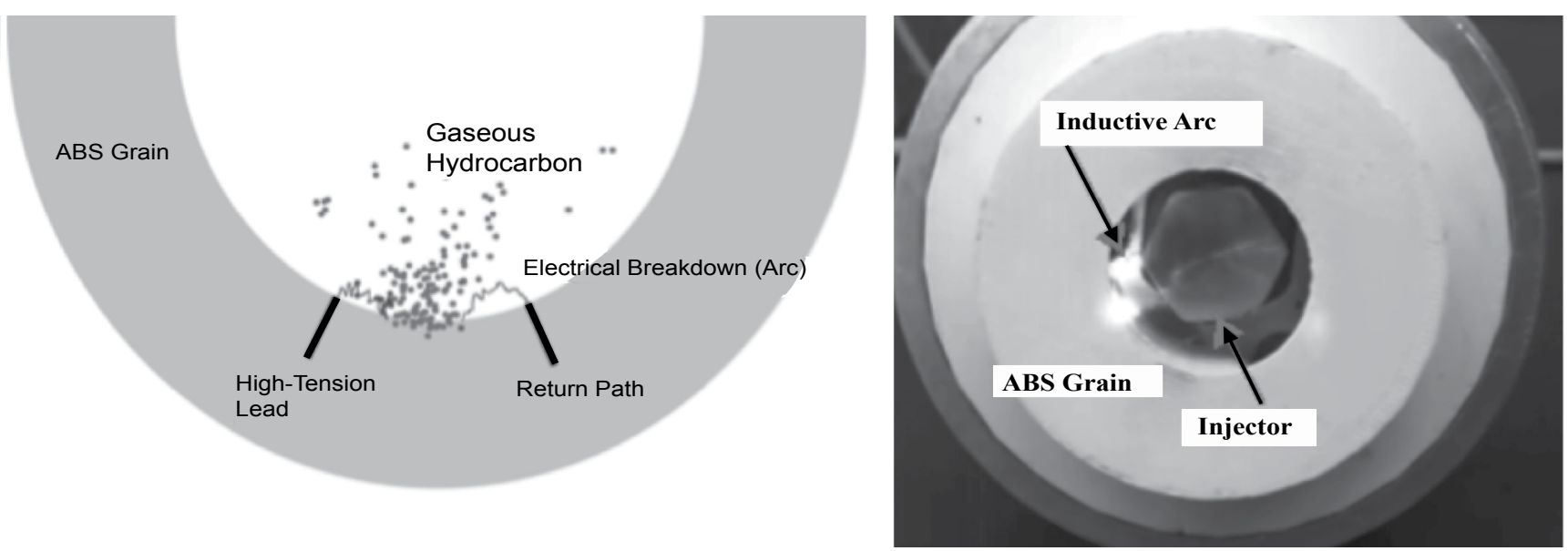

Figure 1: Arc ignitor Joule-Heating concept illustration and demonstration.

3-D printed the components are built-up additively; designs are highly scalable, and internal fuel port can be fabricated with nearly any conceivable geometries.

Arc-Ignition of FDM-processed ABS fuel grains: Finally, FDM-processed ABS possesses unique electrical breakdown properties that can be exploited to allow for rapid on-demand system ignition. Under normal conditions ABS possesses a very high electrical resistivity and dielectric strength and is not considered to be an electrical conductor [22]; however, as FDM-processed ABS is subjected to a moderate electrostatic potential field the layered material structure concentrates minute electrical charges that produce localized arcing between material layers. Joule heating from the resulting arc produces a small but highly-conductive melt layer. This melt layer allows for very strong surface arcing occurring at moderate input voltage levels-between 200 and 300 Volts. Additional Joule heating from the strong surface arcing causes a sufficient fuel material to vaporize to seed combustion when simultaneously combined with an oxidizing flow.

Shortly after this discovery, the authors of this paper made several unsuccessful attempts to reproduce a similar phenomenon with other hybrid fuel materials including HTPB, acrylic, paraffin, and extruded ABS. These experiments also demonstrated that electrical breakdown of FDM-processed ABS occurs at voltages significantly lower than occur with a monolithically fabricated (machined or extruded) article. The extruded or machined ABS does not electrically break down (arc) until voltage levels exceeding 2 kilo-Volts are input across the material. This value is nearly an order of magnitude higher that for a similar FDM-processed test article. Thus, it appears that the observed arcing properties are artifacts of both the grain composite structure due to FDM fabrication and the electromechanical properties of ABS [23].

The discovery of ABS' unique electrical breakdown charac- teristics prompted the invention of an ignition system that takes advantage of the previously described "hydrocarbon seeding" phenomenon. Figure 1 illustrates the top-level concept, where two electrodes are embedded in an ABS fuel grain segment. The layered structure of the FDM-processed ABS provides local surface features of very small radius. The effect is to produce a large ensemble of "electrodes" with a gap-distance on the order of fractions of a millimeter. The inset image shows the electrostatic arc as it moves along the fuel grain surface. Pyrolized gas can also be observed.

The particular orientation of the FDM-processed material layers was found to have a significant effect on the arcing process. In order to qualitatively assess this effect, three conical grains were manufactured; two grains using a Stratasys Dimension 3-D FDM printer [20], and the other grain was machined from extruded ABS stock material. One FDM-processed ignitor grain was printed "vertically-stacked," that is, with deposition layers parallel to the longitudinal axis of the motor and direction of oxidizer flow. The second grain was printed "horizontally-stacked," with deposition layers perpendicular to the longitudinal axis of the motor and perpendicular to the direction of oxidizer flow.

As demonstrated by Whitmore, et al. (2014) [24] after several arc-breakdown cycles, the vertically-stacked ignitor grain ceased to visibly arc. In order evaluate this result the grain cut along the longitudinal axis. Patterns of heavy char indicated that an alternate path between the electrodes had formed, and any circuit closure was occurring internal to the grain surface. Because the loop was closed internal to the grain surface, fuel pyrolysis and the resulting vapor generation did not occur. This behavior was observed for several vertically-stacked test specimens. This behavior did not occur in any of the horizontally-stacked specimens tested, and indicates that horizontal-stacking is the preferred method of manufacture.

Regression rate enhancement using helical fuel 
port structures: Exploiting the FDM fabrication process for ABS offers the potential to revolutionize the manufacture of hybrid rocket fuel grains. FDM can support high production rates and offers the potential of improving hybrid fuel grain quality, consistency, and performance, while reducing development and production costs. These manufacturing advantages are not achievable using the conventional methods of solid propellant production.

Using additive manufacturing, ABS hybrid fuel grains can be fabricated with a large range of port shapes that cannot be produced using conventional casting techniques for fuels like HTPB where tooling must be removed after the material has cured. Of particular interest are helical fuel structures whose centrifugal flow patterns have been shown to significantly increase the fuel regression rate and combustion efficiency $[25,26]$. A summary of results of these helical-port tests, demonstrating regression rate amplification factors exceeding 3.0, are presented in Section IIIC.

\section{System Prototype Test Results}

A series of incremental developmental tests were performed to determine the most appropriate geometry for the arc-ignition fuel grain. References [23,24] describes these developmental experiments in detail. Two fundamental generations of prototypes were built and tested. The first generation ignition system was designed as an external "strap-on" ignitor for an existing $98 \mathrm{~mm}$ diameter hybrid motor. The strap on ignitor replaced the pyrotechnic charges that were previously used for the motor ignition system. The second-generation system reconfigured the strap-on ignitor to move the system inside of the combustion chamber with the ignitor fuel grain section an integral part of the main motor fuel grain.

\section{Second-generation embedded arc-ignitor devel- opment and testing}

The second-generation ignitor concept was developed to overcome multiple practical shortcomings associated with the first-generation prototype strap-on ignitor design. Rather than house a small separate ABS fuel grain mounted on top of the $98 \mathrm{~mm}$ motor cap, channels for conductive paths were built into additively manufactured pre-combustion chambers. These chambers are then "plugged into" a main propellant grain and inserted into the motor tube. For these tests the oxidizer was Gaseous Oxygen (GOX). Later tests successfully replaced GOX with nitrous oxide $\left(\mathrm{N}_{2} \mathrm{O}\right)$ [27]. Vacuum tests were also successfully performed. References [23,24] report on the vacuum test results. References [24,27] report the nitrous oxide test results.

Because the primary objective of this test campaign was to optimize the arc-ignitor fuel grain design, it was deemed too costly and time consuming to manufacture multiple full-scale $98 \mathrm{~mm}$ hybrid fuel grains; thus, as a lowered-cost alternative an existing $98 \mathrm{~mm}$ motor cap previously used for hybrid motor testing was adapted to fit a into a short $10.2 \mathrm{~cm}$ hybrid motor section. Figure 2 shows an exploded view of this developmental unit, dubbed as "Little Joe." The design was engineered such that nozzle geometries could be quickly varied to provide a range of internal chamber pressure conditions. Finally, the GOX injector feed pressure could be modified using a manually adjusted pressure-reducing regulator.

Three different ignitor-grain geometries were evaluated. These configurations were 1) a conical converging section, 2) a stepped-cylindrical section with a flow impingement "shelf," and 3) a stepped-cylindrical section with dual impingement shelves. The first two geometries were evaluated using a straight single port injector and the final dual-shelf geometry was tested using coaxial in-

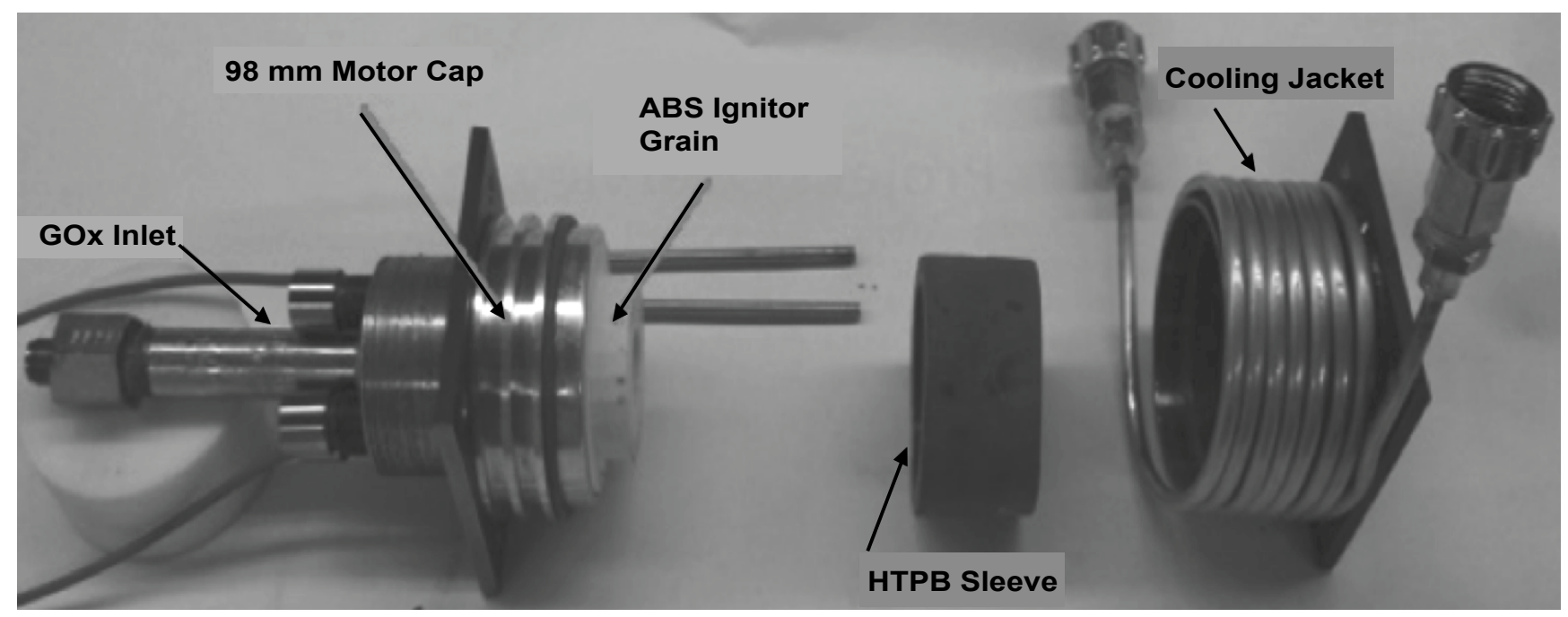

Figure 2: Exploded view of the "Little Joe" ignitor test motor. 


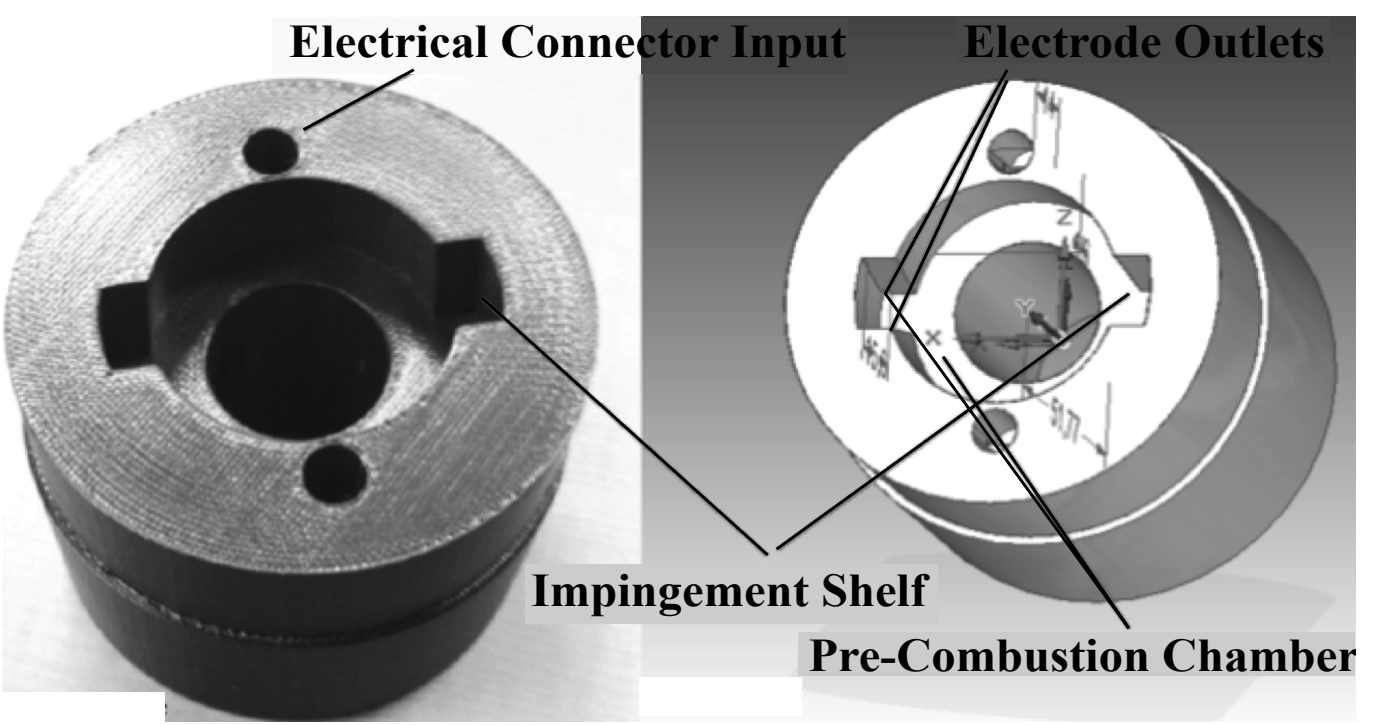

Figure 3: Dual shelf ignitor grain.

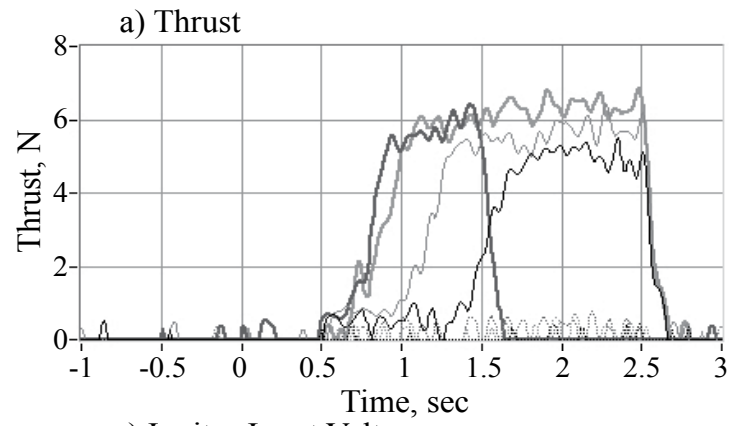

c) Ignitor Input Voltage

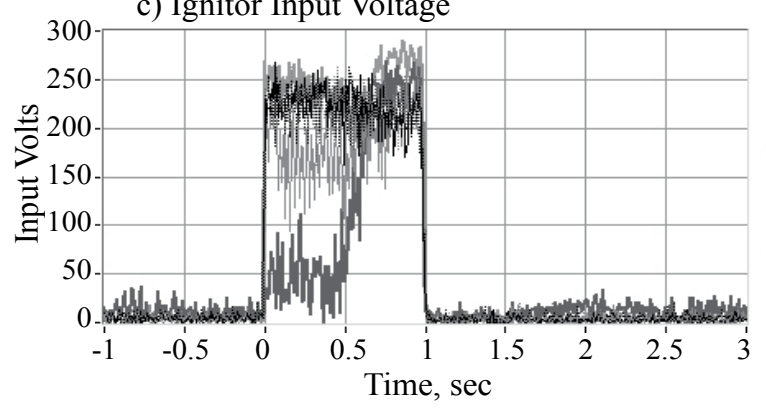

b) Chamber Pressure

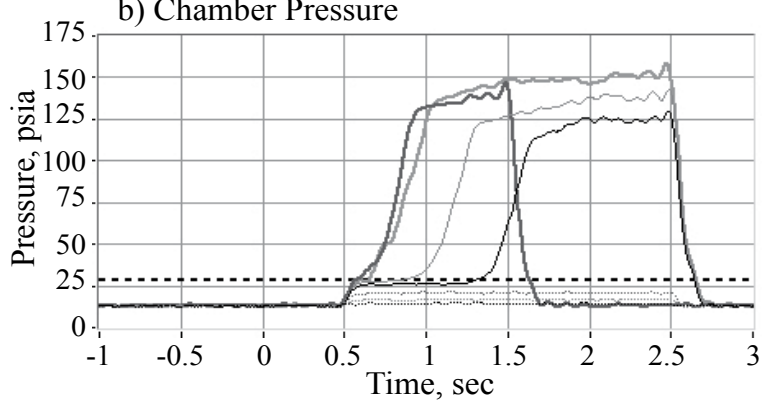

d) Input/Output Power

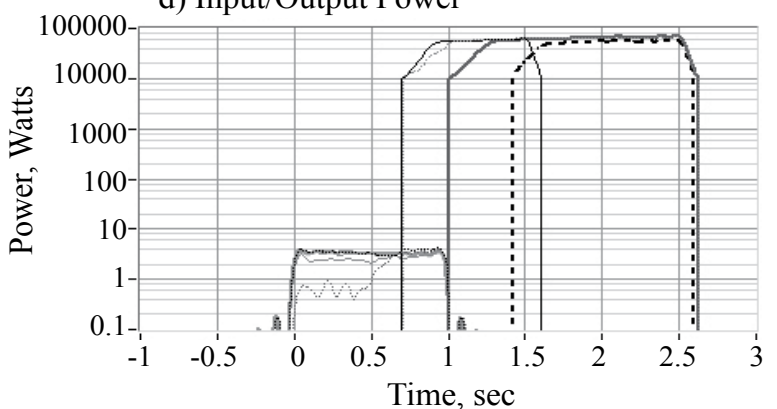

Figure 4: Dual-shelf ignitor, co-axial injector test results.

jector with an axial port, and two side injection ports. During this testing campaign only configuration 3 performed in a reliable and effective manner. Those results are presented in the following paragraphs.

The successful "configuration 3" grain geometry featured a "dual-shelf" design with a coaxial injector comprised of a $0.3175 \mathrm{~cm}$ diameter axial center port, and two $0.15875 \mathrm{~cm}$ (side injection ports. This design directs $2 / 3^{\text {rds }}$ of the oxidizer flow downstream into the combustion chamber and $1 / 3^{\text {rd }}$ of the flow onto the impingement shelves. These features were intended to shorten the combustion latencies exhibited by the earlier configurations. Figure 3 shows the configuration 3 grain design.
Figure 4 presents representative test results from the dual-shelf ignitor grain configuration tests. Here the thrust, chamber pressure, ignition current, and thruster input/ output power time histories are plotted for multiple motor burns. The chamber pressure rises rapidly once a level of approximately 28 psia is achieved, indicating combustion. This pressure represents a minimum oxygen concentration required for ABS combustion, and is also shown plotted on the chamber pressure graph of Figure 4.

At the highest injector inlet pressures (and the resulting chamber pressures) the ignition latencies diminish to less than $10 \mathrm{msec}$. It can be observed that the input power required for ignition is very small-less than 4 


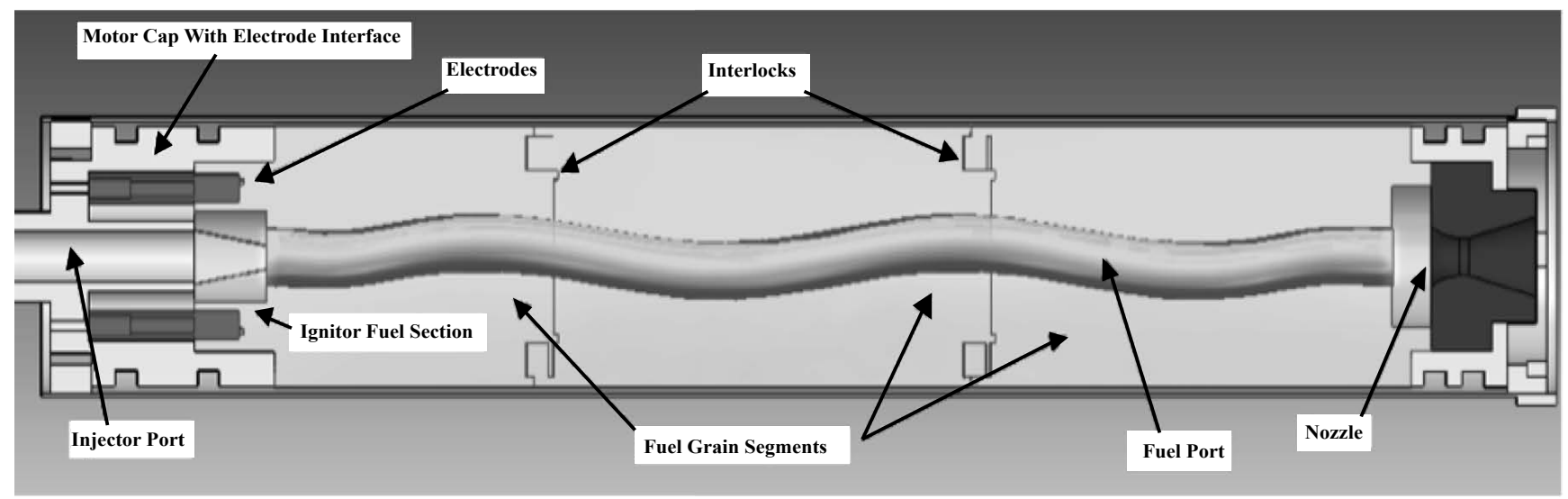

Figure 5: Hybrid motor with integrated ignitor and interlocking fuel grain segments.

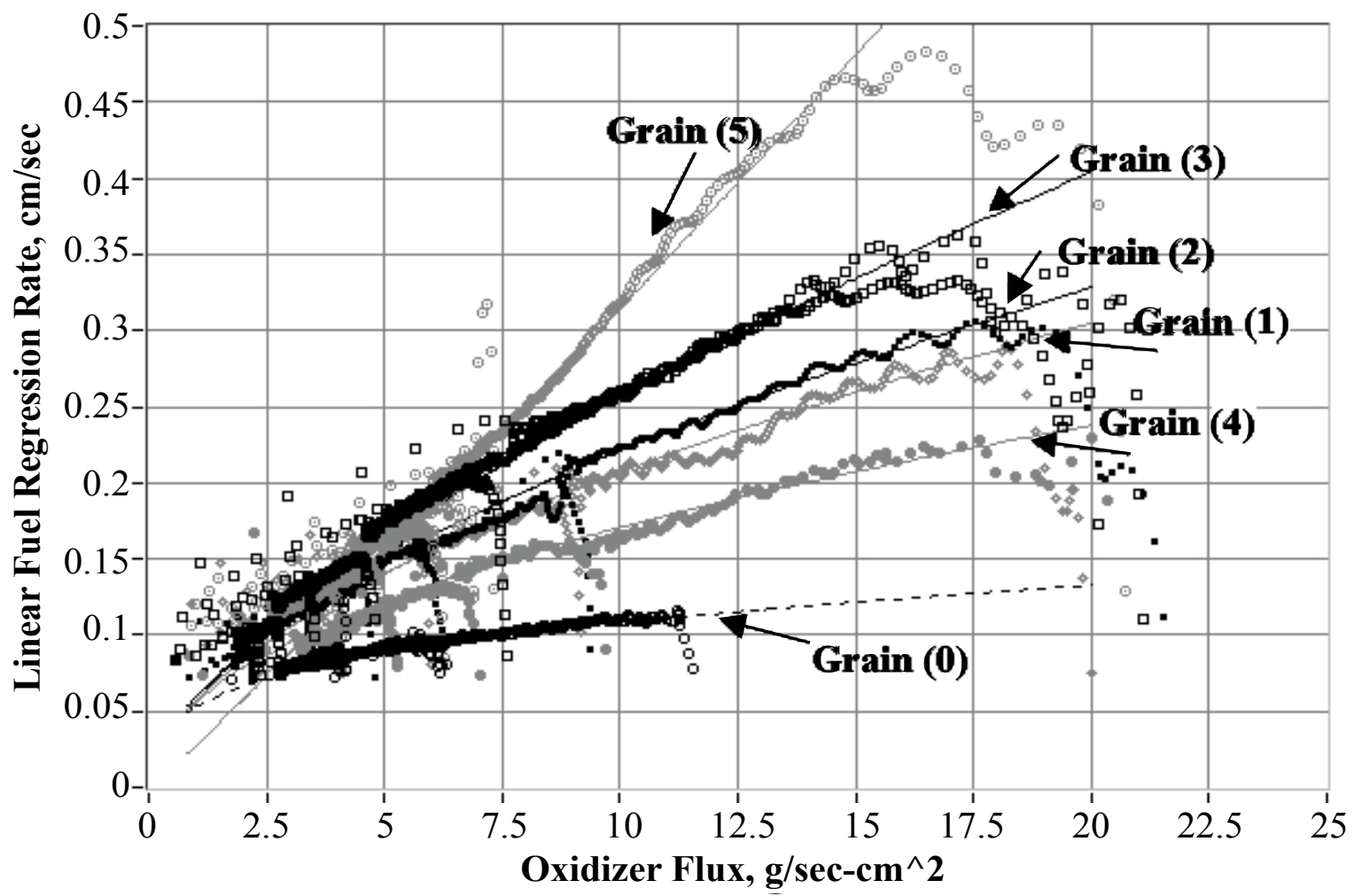

Figure 6: GOX-ABS regression rate comparison for straight-bore and helical grains.

watts-whereas, the generated power exceeds 60 Kilowatts. Thus the ignitor system produces a power amplification factor of greater than 15,000 !

\section{Integrated motor testing on $75 \mathrm{~mm}$ lab-weight prototype}

The GOX/ABS ignition tests were concluded by embedding a scaled version of the "Little Joe" dual-shelf ignitor into the top end of a lab scale $75 \mathrm{~mm}, 170 \mathrm{~N}$ thrust hybrid rocket motor. Figure 5 shows the motor layout with the integrated ignitor section and the helical fuel port. The pistured motor configuration shows an embedded helical structure designed to increase regression rate and combustion efficiency. The design takes advantage of FDM-processing to build the ABS ignitor and fuel grain sections with "snap-together" interlocks that allow individual grain segments to be manufactured separately and then assembled for combustion.

Figure 6 compares typical thrust, chamber pressure, ignitor current, and input power time histories for the 3 consecutive "hands off" burns of the $75 \mathrm{~mm}$ motor with the embedded ignitor grain. The burn profiles exhibited excellent run-to-run consistency with low deviations in both thrust and chamber pressure. Also the response fidelity of the integrated system is consistently greater than was obtained with the cobbled-together "Little-Joe" test apparatus, indicating that the additional chamber volume downstream of the ignitor section has little effect upon the ignition kinetics. 
Table 1: Fuel grain gemetries for helix regression tests.

\section{Grain Fuel port parameters}

\begin{tabular}{|l|l|l|l|l|l|l|} 
no. & Port length, $\mathrm{cm}(L)$ & $\begin{array}{l}\text { Pitch length, } \mathrm{cm} \\
(P)\end{array}$ & $\begin{array}{l}\text { Initial port diameter, } \\
\mathrm{cm}\left(D_{0}\right)\end{array}$ & $\begin{array}{l}\text { Initial helix diameter, } \mathrm{cm} \\
(d)\end{array}$ & Pitch ratio, N ${ }^{*} d / D$ & No. of burns \\
\hline 0 & 35.98 & 15.24 & 1.524 & -- & -- & 3 \\
\hline 1 & 35.98 & 15.24 & 1.524 & 0.762 & 1.177 & 5 \\
\hline 2 & 35.98 & 15.24 & 1.524 & 1.143 & 1.77 & 5 \\
\hline 3 & 35.98 & 15.24 & 1.524 & 1.524 & 2.361 & 6 \\
\hline 4 & 22.86 & 15.24 & 1.524 & 0.762 & 0.75 & 5 \\
\hline 5 & 22.86 & 2.7 & 1.524 & 1.143 & 6.35 & 6 \\
\hline
\end{tabular}

For these tests, the motor featured a semi-optimized nozzle with an expansion ratio of 3.5. The mean delivered Isp at ambient conditions was 228.7 seconds. This delivered specific impulse extrapolates to a vacuum Isp of greater than 300 seconds using a 20:1 expansion ratio nozzle-significantly higher than can be achieved by a hydrazine-based system.

\section{Regression rate enhancement with helical port structures}

At the conclusion of the ignition test series the 75 $\mathrm{mm}$ motor was adapted to investigate the effects of using different helical port structures to enhance the fuel regression rates. References $[25,26]$ present detailed descriptions of the test apparatus and complete test results. It is well known that helical pipe flows have the effect of significantly increasing the local skin friction coefficient. Helical flows also introduce a centrifugal component into the flow field. In hybrid rocket applications, this centrifugal component has the effect of thinning the wall boundary layer-bringing the flame zone closer to the wall surface and increasing the flame diffusion efficiency.

The helical structure is defined by three parameters, 1) the nominal fuel port diameter $D_{0}$, the helix loop diameter $d$, and the helix pitch length, $P$. The pitch length is defined as the distance between the centerlines of two consecutive helical wraps, where $\mathrm{L}$ is the port length and $\mathrm{N}$ is the number of rotations of the helical centerline,

$$
p=L / N
$$

A series of tests were performed to evaluate the effectiveness of helical fuel port structures in increasing the fuel regressin rate. For this series of tests the motor configuration of Figure 5 was used, and the test fuel grains were manufactured simultaneously as interlocking segments on a Stratasys Dimension 1200es 3-D FDM printer [28] from standard density $(0.975 \mathrm{~g} / \mathrm{cm} 3)$ ABS stock material. Joints were bonded using commercial grade ABS pipe joint cement. Six fuel grain geometries, a straight bore-cylindrical grain and five helical ports with varying helix geometries, were tested. In all 31 static firings were performed as a part of this testing campaign.

The first guel grain (0) tested had a cylindrical port and established the baseline for the regression rate of the propellants. The next three grains, (1), (2), and (3) tested featured a total grain length of $35.98 \mathrm{~cm}$ with approximately 2.5 turns along the length of the fuel port. Excluding the post combustion chamber, the resulting pitch distance was $15.24 \mathrm{~cm}$. The final two grains investigated the effects of both total fuel grain length and pitch distance on the regression rate. Grain (4) was identical for grain (1) except that is was truncated at $22.86 \mathrm{~cm}$ length. The final grain (5) was also truncated at $22.85 \mathrm{~cm}$ length, but featured 8.5 total rotations, and a pitch length of $2.7 \mathrm{~cm}$. Table 1 summarizes the parameters of these fuel grains. Column 6 of this table also lists the helical "pitch ratio"; defined as the ratio of the initial helix loop diameter multiplied by the number of loops divided by the initial fuel port diameter,

$$
{ }_{R}=\frac{L}{P} \cdot \frac{d}{D_{0}}=\mathrm{N} \cdot \frac{d}{D_{0}}
$$

Although real time thrust-stand oxidizer massflow and motor mass measurements were obtained; for this testing campaign each grain was burned multiple times, and the motor disassembled after each test to allow intermediate mass measurements as a check on the accuracy of the real-time measurements.

The fuel regression rate was calculated from the differences between the measured oxidizer and nozzle exit mass flows according to Equation (3). In Equation (3) is the mean fuel port diameter calculated by simultaneously integrating the rate of regression.

$$
\overline{\dot{r}}=\frac{\dot{m}_{\text {fuel }}}{2 \cdot \rho_{\text {fuel }} \cdot \pi \cdot \bar{r} \cdot L}=\frac{\dot{m}_{\text {total }}-\dot{m}_{o x}}{2 \cdot \rho_{f u e l} \cdot \pi \cdot \bar{r} \cdot L .}
$$

Integrating Equation (3) from the initial condition to the current time solves for the longitudinal mean of the instantaneous fuel port diameter

$$
\bar{r}(t)=\sqrt{r_{0}^{2}}+\int_{0}^{t} \frac{\dot{m}_{\text {fuel }} \cdot d t}{\rho_{\text {fuel }} \cdot \pi \cdot L .}
$$

Using estimated fuel port radius and the measured oxidizer massflow rate, the mean longitudinal oxidizer massflux is estimated as 


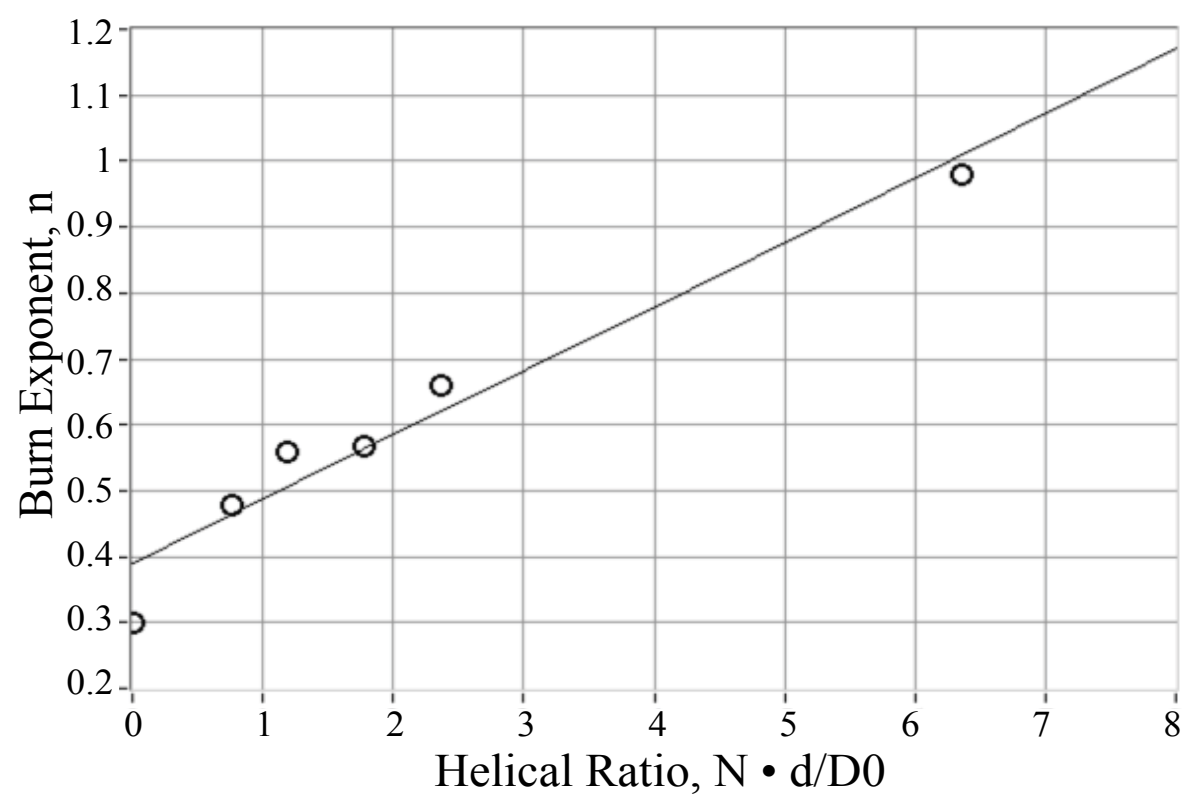

Figure 7: Curve fit exponent plotted against helical pitch ratio.

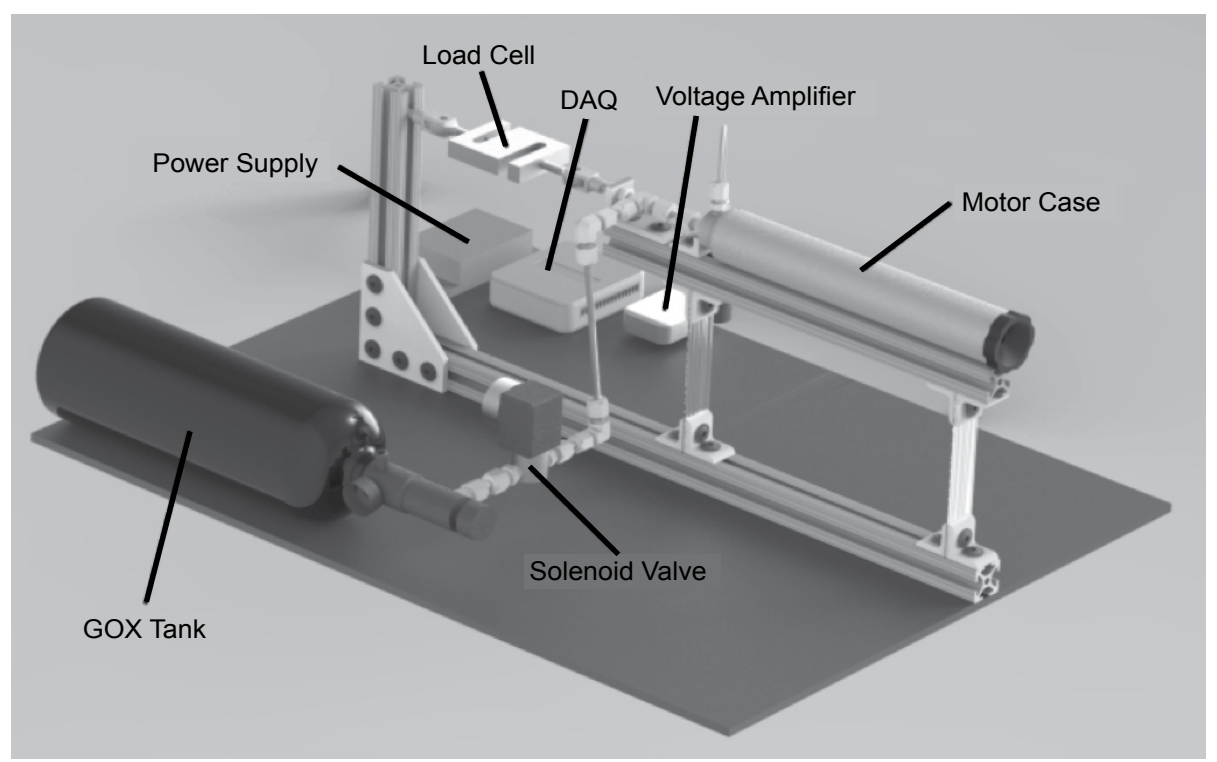

Figure 8: CAD drawing of flight-weight integrated system.

$$
\bar{G}_{o x}=\frac{\dot{m}_{o x}}{\pi \cdot \mathrm{r}_{(t)}^{2}}
$$

Figure 7 plots the fuel regression rates as a function of the longitudinal mean of the oxidizer massflux. Also plotted for each fuel grain are exponential curve fits of the form

$$
\dot{r}=\mathrm{a} \cdot \mathrm{G}_{o x}^{n}
$$

When compared to the cylindrical fuel port grain, all of the helical grains exhibit a significant increase in the mean regression rate Qualitatively, it is reasonable to infer that the helical loop diameter $(d)$, pitch distance $(P)$, the Initial fuel port diameter $\left(D_{0}\right)$, and the fuel port length $(L)$ all have distinct effects on the resulting regression rate profile. The overall correlation is established by plotting the best-fit burn exponents of Figure 6 against pitch ratio parameter defined by Equation (2). Figure 7 presents these results. The correlation is outstanding.

In Figure 6 the high massflux occurs early in the burn sequences where the fuel port is still quite helical, and the resulting centrifugal flow effects push the flame zone closer to the wall. As the port burns and the massflux drops, the helical structure also regresses both radically and longitudinally-gradually becoming more cylindrical in shape. The resulting drop in centrifugal force as the port becomes circular allows pyrolized fuel leaving the grain surface to push the flame zone away from the wall 
and reduces the effects of heat transfer to the walls. The regression rate drops accordingly.

The correlation of Figure 7 explains why the Grain (4) curve appears to be "out of place" on Figure 6. Even though the helical parameters of Grain (4) are identical to those of Grain (1), the lower number of turns along the grain length for Grain (4) reduces the overall effectiveness of the helix. Correspondingly, the short pitch length fuel grain where the helical loops are spaced closely together longitudinally shows the highest initial regression rate and most rapid regression rate drop over time.

Finally, because the helical loops in the fuel grain progressively burn together with time, and the fuel port becomes more and more like a cylinder. Conveniently, this effect reduces the potential for excessive unburned fuel "slivers" at the end of the burn lifetime of the fuel grain.

Flight-Weight system development and testing: After proof of concept tests with the ignitor and lab-scale integration tests were completed, an effort was made to scale down the thruster to a flight-weight system to prove the scalability of the system and immediate pulsed re-ig- nitability of the thruster. The previously presented model was used to design a flight-weight unit with a desired $22-\mathrm{N}$ thrust level. Figure 8 presents a schematic of the laboratory apparatus used to test the flight weight system. Figure 9 shows the scaled fuel grain with integrated electrides for the arc-ignition sysem. Figure 10 shows the prorotype flight weight thruster firing.

Figure 11 shows the chamber pressure time history trace measured as the motor was pulse-fired. As shown, the system was successfully restarted 4 times, with startup transients in the range of $\sim 10 \mathrm{msec}$. Over an ensemble of more than 50 tests, and over a wide range of $\mathrm{O} / \mathrm{F}$ ratios, the motor was able to ignite and re-ignite with a high level of probability. The standard-deviation of the burn-to-burn total impulse variability was less than $+5 \%$.

More than 50 test firings were performed for the initial testing campaign of the flight-weight thruster. The resulting specific impulse achieved from this series of ground tests-performed at low altitude, ambient conditions-exceeded 228 seconds for all cases. The resulting standard deviation was approximately $3.5 \%$. The achieved low-altitude $I_{s p}$ value is better than the typical vacuum $\mathrm{I}_{\mathrm{sp}}$

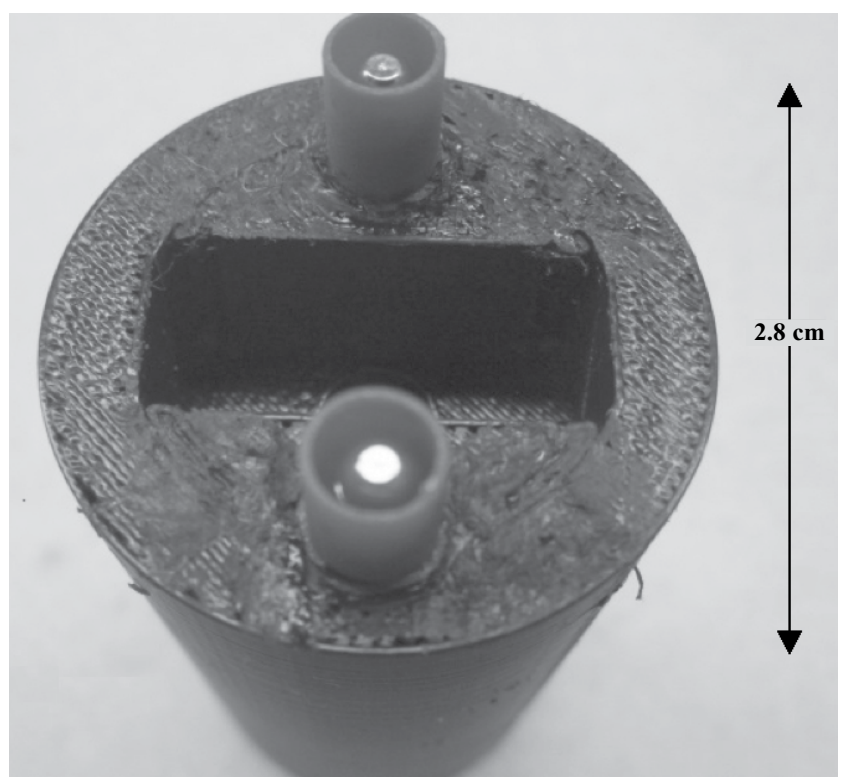

Figure 9: Scaled-Down ABS fuel grain with integrated spark ignitor.

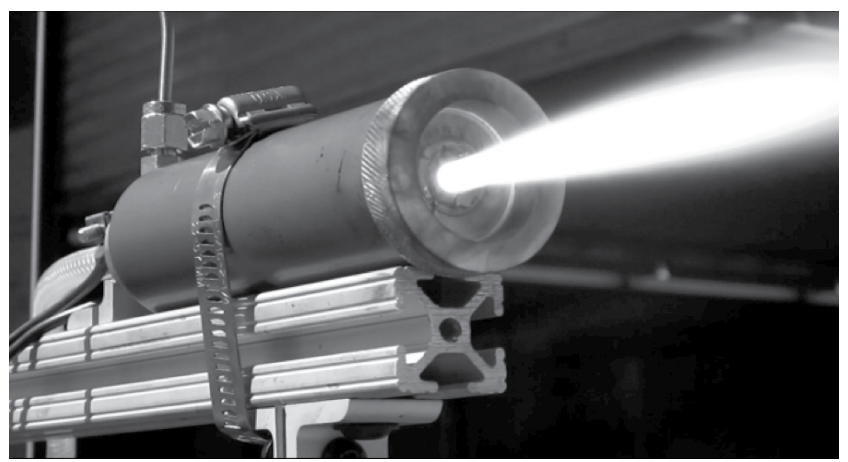

Figure 10: Close-up view of firing flight weight thruster system. 


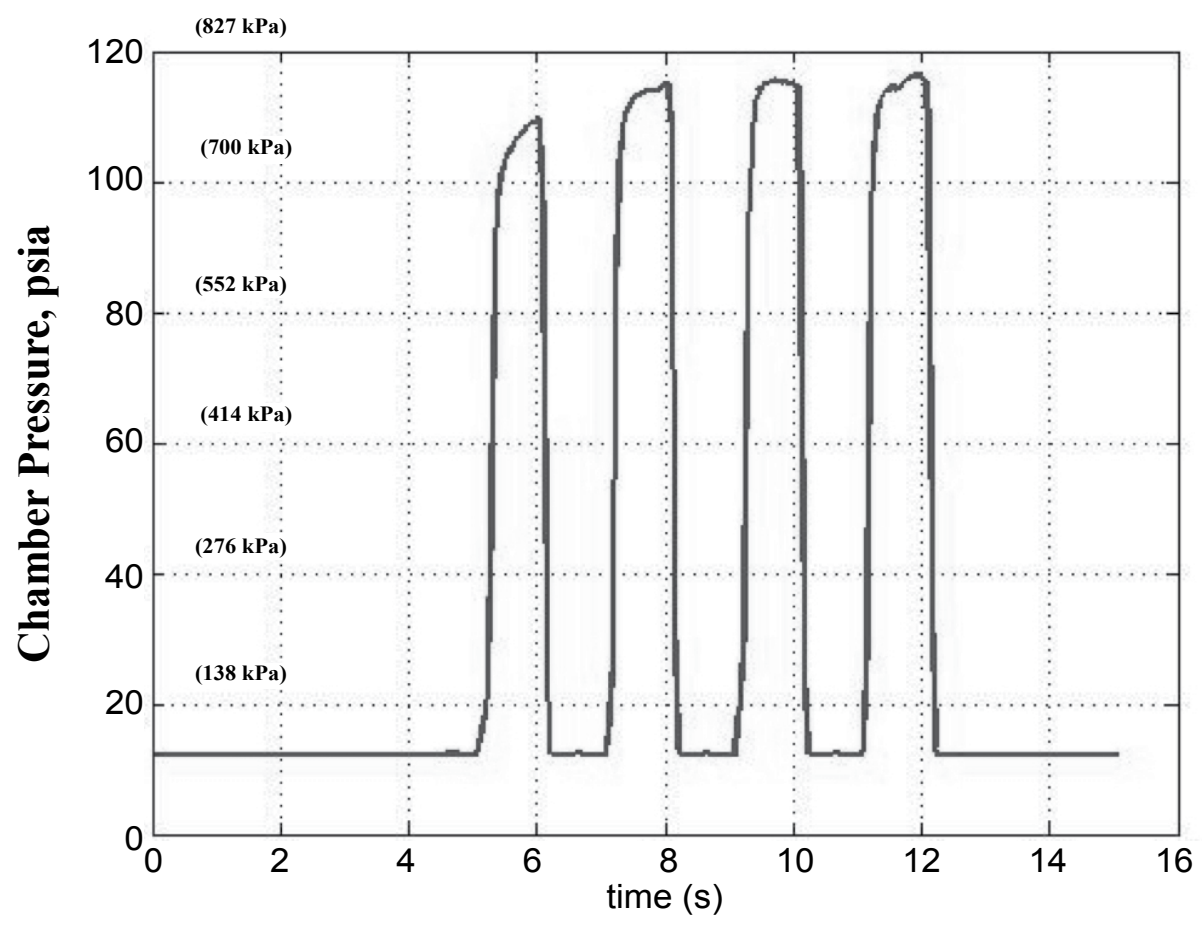

Figure 11: Chamber pressure time history of pulsed burns demonstrating consecutive restart capability.

level of hydrazine of 220 seconds. When the results of the ground tests are extrapolated to vacuum conditions, and assuming an optimized nozzle configuration, the resulting value exceeds 290 seconds-a value that is more than $30 \%$ better.

The development of this flight weight system, along with the results from the previous conceptual/feasibility testing proves the readiness of the additively manufactured grain to be engineered as a "drop-in" replacement for hydrazine, maintaining the consistent restart ability and compact form factor of a hydrazine system, while improving significantly on safety, performance, power-consumption, and cost of materials and operation.

\section{Conclusion}

This paper details the developmental of an innovative "green" hybrid propulsion system using Fused Deposition Modeling (FDM) additive manufacturing. When fully developed this cross-cutting technology offers a wide variety of space propulsion applications, and may allow the development of "drop in" replacements for many existing hydrazine-based propulsion systems. FDM manufacturing circumvents many of the developmental issues normally associated with hybrid rocket systems. FDM manufacturing has allowed the development of multiple prototype hybrid thrusters that exhibit a high degree of restartability, require a low-wattage input for ignition, demonstrate enhanced fuel regression rates, and a offer a highly compact form factor.

After proof of concept tests with the ignitor and lab- weight motor were completed, the developed thruster technology was scaled to flight-weight micro-thrister to prove the scalability of the system technology. This system was used to demonstrate multiple, successive restart capability, with precisely controlled impulse bits. Presented second generation thruster tests results demonstrate a specific impulse performance that is more than $30 \%$ superior to hydrazine-based systems.

Propellants that are $100 \%$ non-toxic, recyclable, and non-detonable achieve the presented specific impulse performance improvement. Because the environmentally benign "green" propellants are mixed only within the combustion chamber once the ignition is initiated, the system is inherently safe and can be piggybacked on a secondary payload without significantly increasing overall mission risk to the primary payload. The ease of handling when compared to hydrazine means that systems could be loaded into spacecraft faster and more cheaply because launch-pad workers don't have to wear cumbersome full-body protection. Green propellants probably won't completely replace hydrazine, the workhorse of many research satellites and interplanetary missions. Nor will they replace the powerful fuels typically used to launch rockets. But the new printed fuels will enable cheaper and more flexible mission designs.

\section{References}

1. King SM, Marx PC, Taylor D (1969) The Aerospace Corporation Shell 405 Catalyst Evaluation Program, Volume I: Physical and Catalytic Properties. Aerospace Report No TR-066 (5210-10) 1. 
2. Sutton GP, Biblarz O (2008) Rocket Propulsion Elements. ( $8^{\text {th }}$ edn), Wiley Interscience, New York, 263-265.

3. Choudhary G, Ilansen H, Donkin S, Kirman C (1997) Toxicological Profile for Hydrazines. US Department of Health and Human Services Public Health Service Agency for Toxic Substances and Disease Registry. Atlanta, 1-224.

4. DeSain John D (2011) Green Propulsion: Trends and Perspectives.

5. Goldstein, Edward (2012) The Greening of Satellite Propulsion. Aerospace America, 26-28.

6. Bombelli V (2003) Economic Benefits for the Use of Non-toxic Monopropellants for Spacecraft Applications. Joint Propulsion Conference and Exhibit Huntsville, Alabama.

7. Haeseler D, Bombelli V, Vuillermoz P, Lo R, Marée T, et al. (2004) Green Propellant Propulsion Concepts for Space Transportation and Technology Development Needs. Proceedings of the 2nd International Conference on Green Propellants for Space Propulsion, Italy.

8. Pokrupa N, Anglo K, Svensson O (2011) Spacecraft System Level Design with Regards to Incorporation of a New Green Propulsion System. 46th AIAA/ASME/SAE/ASEE Joint Propulsion Conference and Exhibit, Sandiego.

9. Nagamachi MY, Oliveira JI, Kawamoto AM, Dutra RC (2009) ADN - The new oxidizer around the corner for an environmentally friendly smokeless propellant. Journal of Aerospace Technology and Management 1: 153-160.

10. Venkatachalam S, Santhosh G, Ninan KN (2004) An Overview on the Synthetic Routes and Properties of Ammonium Dinitramide (ADN) and other Dinitramide Salts. Propellants Explosives Pyrotechnics 29: 178-187.

11. Rheingold AL, Cronin JT, Brill TB, Ross ZK (1987) Structure of Hydroxylammonium Nitrate (HAN) and the Deuterium Homolog. Acta Crystallographica 43: 402-404.

12. Hawkins TW, Brand AJ, McKay MB, Tinnirello M (2010) Reduced Toxicity, High performance Monopropellant at the U.S. Air Force research Laboratory. 4th International Association for the Advancement of Space Safety Conference, Huntsville, Alabama.

13. Meinhart D (1988) Selection of Alternate Fuels for HANBASED Monopropellants. 27th JANAFF PDCS and 16th S\&EPS Joint Meeting, 674: 143-147.

14. Persson M, Anflo K, Dinardi A (2012) A Family of Thrusters For ADN-Based Monopropellant LMP-103S. AIAA-20123815, 48th AIAA/ASME/SAE/ASEE Joint Propulsion Conference \& Exhibit, Atlanta, Georgia.

15. Spores RA, Masse R, Kimbrel S (2013) GPIM AF-M315E Propulsion System. $49^{\text {th }}$ AIAA/ASME/SAE/ASEE Joint Propulsion Conference, San Jose, California.
16. Katsumi T, Hori K (2010) Combustion Wave Structure of Hydroxyl ammonium Nitrate Aqueous Solutions. 46th AIAA/ ASME/SAE/ASEE Joint Propulsion Conference \& Exhibit, Nashville, Tennessee.

17. Anon (1988) Hazard Analysis of Commercial Space Transportation. Volume 1: Operations; Volume 2: Hazards; volume 3: Risk Analysis. US Dept of Transportation, 182.

18. Cheng CG, Farmer RC, Jones HS, McFarlane JS (1994) Numerical Simulation of the Internal Ballistics of a Hybrid Rocket. 30th AIAA/ASME /SAE/ASEE Joint Propulsion Conference \& Exhibit, Indianapolis.

19. Anon (2010) Department of Defense Interface Standard, Eletromagnetic Environmental Effects Requirements for Systems.

20. Anon (2014) Weapons Systems and Platforms (WP) Focus Area. Environmentally Friendly Sustainable Binder System for Energetic Materials. 2014 Strategic Environmental Research and Development Program (SERDP), Exploratory Development (SEED) BAA-13-0002. This proposal responds specifically to FY2014 SEED Statement of Need, WPSEED-14-01.

21. Whitmore Stephen A, Peterson Zachary W, Eilers Shannon D (2013) Comparing Hydroxyl Terminated Polybutadiene and Acrylonitrile Butadiene Styrene as Hybrid Rocket Fuels. Journal of Propulsion and Power 29: 582-592.

22. Shugg WT (1986) Handbook of Electrical and Insulating Materials. Van Nostrand Reinhold, New York.

23. Whitmore SA, Inkley NR, Merkley DP (2014) Development of a Power Efficient, Restart-Capable Arc Ignitor for Hybrid Rockets. 50th AIAA/ASME/SAE/ASEE Joint Propulsion Conference, Cleveland, Ohio.

24. Whitmore Stephen A, Inkley Nathan R, Merkley Daniel P, Judson Michael I (2015) Development of a Power-Efficient, Restart-Capable Arc Ignitor for Hybrid Rockets. Journal of Propulsion and Power 31: 1739-1749.

25. Whitmore Stephen A, Walker Sean D, Merklely Daniel P, Mansour Sobbi (2015) High Regression Rate Hybrid Rocket Fuel Grains with Helical Port Structures. Journal of Propulsion and Power 31: 1727-1738.

26. Whitmore Stephen A, Walker Sean D, Merklely Daniel P (2015) Engineering Model for Hybrid Rocket Regression Rate Amplification by Helical Fuel Ports. 51st AIAA/SAE/ ASEE Joint Propulsion Conference, Orlando, Florida.

27. Whitmore Stephen A (2015) Additively Manufactured Acrylonitrile-Butadiene-Styrene-Nitrous-Oxide Hybrid Rocket Motor with Electrostatic Igniter. Journal of Propulsion and Power 31: 1217-1220.

28. Anon (2013) Dimension 1200es, Durability Meets Affordability. 